This is the author's copy of the publication as archived in the DLR electronic library at http://elib.dlr.de. Please consult the original publication for citation, see https://arc.aiaa.org/doi/abs/10.2514/6.2022-0263.

\title{
Dynamic Modeling and Analysis of Tilt-Wing Electric Vertical Take-Off and Landing Vehicles
}

\author{
Marc S. May and Daniel Milz and Gertjan Looye
}

Electric vertical take-off and landing (eVTOL) aircraft enable new transport options in regional and urban air mobility. One promising but only little investigated and understood subcategory comprises tilt-wing eVTOLs. They offer high efficiency and long flight ranges but come with a trade-off in increased complexity. Consequently, a critical step towards market entry is the development of mature and safe hybrid pilot-autonomy control systems, including fault detection, identification, and recovery (FDIR) concepts. That requires a mid-fidelity dynamic model with sufficient accuracy, which is not yet available despite a long history of tilt-wing research. Without a representative model, no detailed analysis and identification of a trimmed transition trajectory could be performed. This, however, is a crucial step in the development of a control system. We approach the problem by applying and combining current modeling approaches. Furthermore, a trim analysis of different flight phases, including the transition, is conducted. The identified model lays the foundation for a representative and detailed development and investigation of future control designs, bringing tilt-wing eVTOLs closer to airworthiness.

\begin{tabular}{|l|}
\hline \multicolumn{1}{c|}{ Copyright Notice } \\
\hline $\begin{array}{l}\text { Copyright @ } 2022 \text { by German Aerospace Center (DLR). Published by the American } \\
\text { Institute of Aeronautics and Astronautics, Inc., with permission. }\end{array}$ \\
\hline $\begin{array}{l}\text { May, Marc S. and Milz, Daniel and Looye, Gertjan (2022) "Dynamic Modeling and Analysis } \\
\text { of Tilt-Wing Electric Vertical Take-Off and Landing Vehicles," AIAA 2022-0263. AIAA } \\
\text { SCITECH } 2022 \text { Forum. January 2022. }\end{array}$
\end{tabular}




\title{
Dynamic Modeling and Analysis of Tilt-Wing Electric Vertical Take-Off and Landing Vehicles
}

\author{
Marc May*, Daniel Milz ${ }^{\dagger}$ and Gertjan Looye \\ Institute of System Dynamics and Control, German Aerospace Center (DLR), 82234 Weßling, Germany
}

\begin{abstract}
Electric vertical take-off and landing (eVTOL) aircraft enable new transport options in regional and urban air mobility. One promising but only little investigated and understood subcategory comprises tilt-wing eVTOLs. They offer high efficiency and long flight ranges but come with a trade-off in increased complexity. Consequently, a critical step towards market entry is the development of mature and safe hybrid pilot-autonomy control systems, including fault detection, identification, and recovery (FDIR) concepts. That requires a mid-fidelity dynamic model with sufficient accuracy, which is not yet available despite a long history of tilt-wing research. Without a representative model, no detailed analysis and identification of a trimmed transition trajectory could be performed. This, however, is a crucial step in the development of a control system. We approach the problem by applying and combining current modeling approaches. Furthermore, a trim analysis of different flight phases, including the transition, is conducted. The identified model lays the foundation for a representative and detailed development and investigation of future control designs, bringing tilt-wing eVTOLs closer to airworthiness.
\end{abstract}

\section{Introduction}

$\mathrm{T}$ HE steady development of mobility in the last centuries made distances appear smaller over time and permits our present globalized way of life. However, the growing mobility demand seems to reach saturation despite high efforts to improve and expand the traffic system. In 2010, around 6.4 trillion euros were spent worldwide on peoples' and goods' mobility [1]. These almost 1000 euros per person globally account for $13 \%$ of the global gross domestic product (GDP). The mobility market has quadrupled over the last 40 years corresponding to an annual growth rate of $3.8 \%$ [1], which exceeds the world's average annual GDP growth of $3.1 \%$ [1]. The increased demand caused by a growing world population and a steadily rising global prosperity is especially notable in urban areas. As a consequence of urbanization, sparsely populated areas are often neglected and thus lose touch with the economic and cultural centers [2, 3]. Besides an efficient deployment of conventional modes of transportation, new mobility concepts enabled by modern technology are required to face these problems. Urban and regional air mobility is a disruptive approach, shifting short- and medium-distance transport into the air by deploying electric vertical take-off and landing vehicles (eVTOLs). Aircraft capable of vertical take-off and landing (VTOL) have been part of aeronautical research for decades [4]. However, except for helicopters and some special, mostly military configurations, VTOL aircraft have not made it to the market yet. Vectored thrust concepts are a promising type of eVTOL where all thrusters are used for both hover and cruise. An advantage of this vehicle class is its flexibility to perform vertical and short take-offs and landings while maintaining efficiency during cruise flights. Tilt-wing aircraft are particularly characterized by the latter advantage [5]. A significant modification of the rotor wake and accompanying performance losses are avoided by tilting both the propulsion system and wing [6,7]. Consequently, a good compromise of hover and cruise flight efficiency can be achieved. Thus, compared to other VTOL systems like multicopters or lift+cruise configurations, tilt-wing VTOL aircraft feature higher airspeed and extended flight ranges in a trade-off with mechanical complexity and complex flight control systems [5, 8, 9].

Due to the mentioned characteristics, tilt-wing eVTOL are especially suited to offer mobility options for connections where the required extension of traffic infrastructure would be expensive or unfeasible. Regarding their complexity, one important step towards market entry is the development of mature and safe hybrid pilot-autonomy control systems [10] incorporating fault detection, identification, and recovery (FDIR) concepts. However, a representative dynamic model is required to investigate suitable control and FDIR concepts, compare them, and raise their technology readiness level. Although multiple tilt-wing eVTOL concepts show successful test flights [11-13], and tilt-wing VTOLs were part of

\footnotetext{
*Research Assistant, marc.may@dlr.de both authors contributed equally to this work

${ }^{\dagger}$ Research Assistant, daniel.milz@dlr.de both authors contributed equally to this work

${ }^{\ddagger}$ Head of Department, gertjan.looye@dlr.de. AIAA member
} 
research and prototype development for decades, it still lacks a publicly available and representative dynamic model for control and FDIR design. Nevertheless, the complexity of the dynamic model shall be limited to enable fast and efficient design cycles. Thus, a mid-fidelity model offering medium-complexity, fast simulation cycles, and physical transparency while covering all relevant dynamic effects is desirable. Complex aerodynamic effects of the tilting, rotor-wing, and rotor-rotor interactions make defining such a model especially hard. Additionally, no detailed model analysis and identification of a trimmed transition trajectory could be performed due to the lack of a mid-fidelity model. However, isolated flight and wind tunnel test results already provide trim states for specific flight conditions [14, 15].

This research lays the foundation for the development of a new mid-fidelity model of a tandem tilt-wing eVTOL by summarizing current modeling approaches, combining and applying them to the exemplary vehicle Airbus $A^{3}$ Vahana. The model should represent all important phenomena on tilt-wing eVTOL aircraft. These include the general flight mechanics, electric propulsion system, and general aerodynamic effects. However, the complex aerodynamics during the transition phase is hard to model and not yet thoroughly investigated [4]. Therefore, these phenomena are approximated in a first approach to cover the main effects, although a mid-fidelity aerodynamic model is intended. Still, important insights for future work on modeling, control, and FDIR design can be drawn from a trim study of the obtained model over the whole flight envelope.

A short overview of the content of this paper shall be given in the following. First, the configuration is defined in Section II Sections $\Pi$ III $\mathrm{V}$ then introduce the flight mechanic, aerodynamic, and propulsive model. Afterward, Section VI includes a model analysis and trim study. The discussion and conclusion finally take place in SectionVII]

\section{Aircraft Configuration}

This paper on eVTOL dynamic modeling shall lay the foundation for future research on flight control and FDIR. The eVTOL design is consequently out of the scope of this work, and it is appropriate to choose a reference design that has already been evaluated. Tandem tilt-wing vehicles currently have a low technology readiness level since they are the most complex vehicle configuration for eVTOL. Therefore, they are relatively unpopular in the emerging industry, which is initially focused on a quick market entry. Still, with the NASA LA-8 and the Airbus $A^{3}$ Vahana, two popular testbeds exist that can be referred to. Both are investigated in several publications [16, 17]. The aerodynamic data model derived from wind tunnel experiments for the NASA LA-8 [16] is particularly interesting from a modeling perspective. Nevertheless, the Airbus $A^{3}$ Vahana has the advantage of an open-source design study * while being the only tandem tilt-wing concept with a prototype that accomplished above 100 test flights In terms of aerodynamics, the open-source tool DUST for particle wake simulation was developed in collaboration with Airbus $A^{3}$ and validated for the Vahana [17], see Section IV] Consequently, the Vahana design was chosen as a reference for the presented modeling approach.

The aircraft and its relevant dimensions, derived from different images and sketches of the vehicle, are shown in Figure 1 . As presented in the isometric view, a total of 22 actuators can be deployed by the flight control system (FCS) to manipulate the aircraft's motion. Two uncoupled tilt actuators can deflect the two airfoils and attached propulsion systems by tilt angle $\delta_{\mathrm{W} 1}$ for the canard and $\delta_{\mathrm{W} 2}$ for the main wing to allow transitional flight. The propulsive forces and

moments of the eight rotors are controlled by their individual rotor speeds $\omega_{1} \ldots \omega_{8}$ and blade pitch angles $\theta_{1} \ldots \theta_{8}$. Above that, 4 control surfaces with deflections $\eta_{1} \ldots \eta_{4}$ and 2 static vertical stabilizers at the tips of the main wing are provided for aerodynamic control and stabilization.

*E.g., https://github.com/VahanaOpenSource https://acubed.airbus.com/blog/vahana/vahana-configuration-tradestudy-part-i/ and https://acubed.airbus.com/blog/vahana/vahana-design-process-part-i-putting-pen-to-paper/[ [Accessed: June 2021]

https://evtol.com/news/airbus-vahana-evtol-concludes-flight-testing/[Accessed: June 2021] 

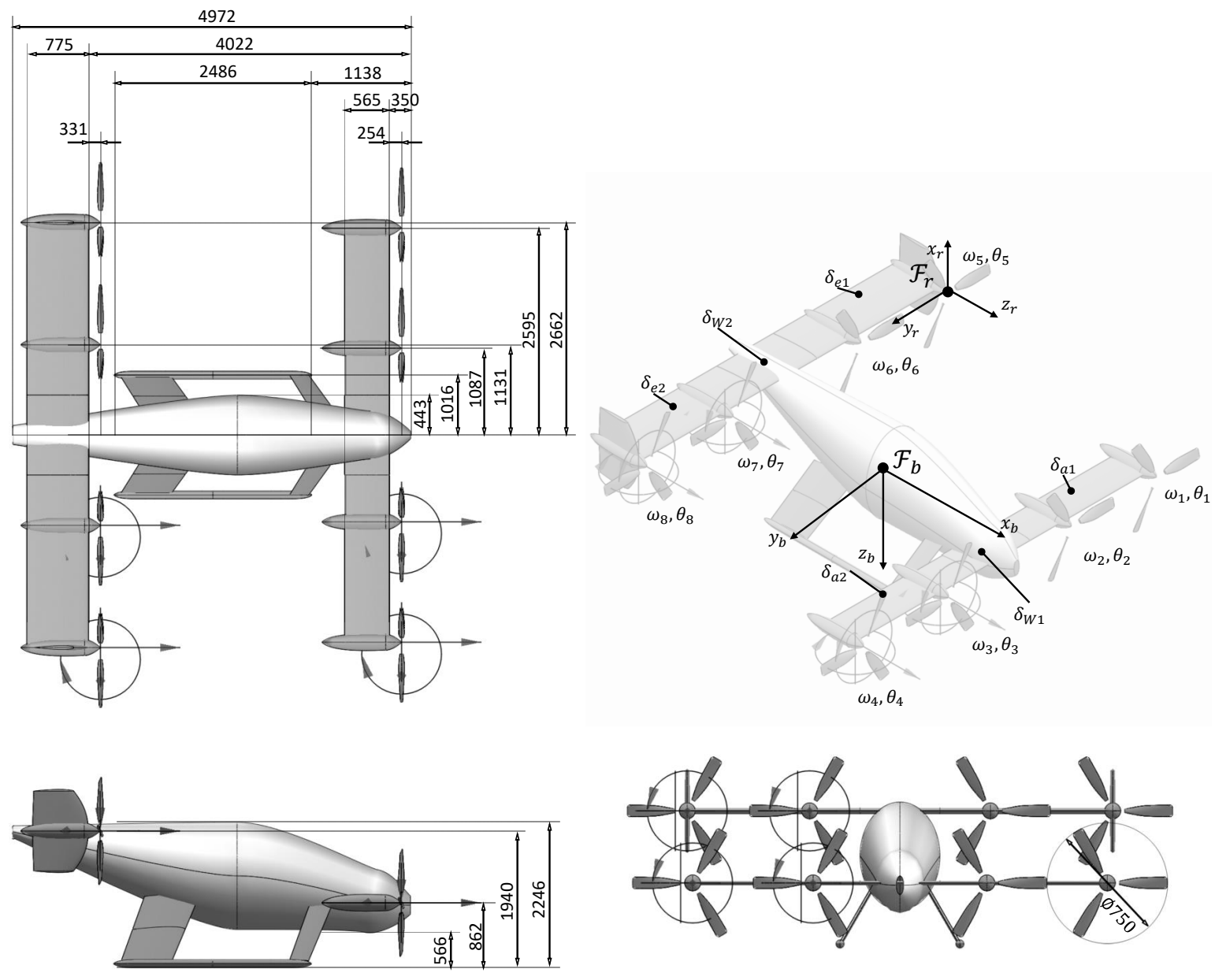

Fig. 1 Geometry and actuation of the Airbus $A^{3}$ Vahana, visualized in the OpenVSP software [18].

\section{Flight Mechanics Model}

It is sufficient to describe the aircraft movement in flat earth coordinates when considering the relatively short ranges, low velocities, and low altitudes of eVTOL missions [19]. Consequently, the general relation of the body $(b)$ angular rates

$$
\boldsymbol{\Omega}^{i b}=\boldsymbol{\Omega}^{i e}+\mathbf{\Omega}^{e n}+\boldsymbol{\Omega}^{n b}=\mathbf{\Omega}^{n b}=\mathbf{\Omega}^{b}
$$

since the rotations between inertial frame $\mathcal{F}_{\mathrm{i}}$ and ECEF frame $\mathcal{F}_{\mathrm{e}}$ frame as well as ECEF and NED frame $\mathcal{F}_{\mathrm{n}}$ frame are both zero. $\mathcal{F}_{\mathrm{n}}$ frame fulfills the characteristics of an inertial frame. The derivative of the Euler angles is $\boldsymbol{\Theta}=[\phi, \theta, \psi]^{T}$ is found as

$$
\dot{\boldsymbol{\Theta}}=R_{\Theta \mathrm{b}} \cdot \mathbf{\Omega}^{b}
$$

with

$$
R_{\Theta \mathrm{b}}=\left[\begin{array}{ccc}
1 & \sin \phi \tan \theta & \cos \phi \tan \theta \\
0 & \cos \phi & -\sin \phi \\
0 & \frac{\sin \phi}{\cos \theta} & \frac{\cos \phi}{\cos \theta}
\end{array}\right]
$$

The Euler angles relate the body attitude to frame $\mathcal{F}_{\mathrm{n}}$ and are thus deployed for transforming the local body velocity ${ }^{\mathrm{b}} \mathbf{v}^{n b}$ into the inertial frame, thereby allowing to describe the motion relative to a reference point 0 on the flat earth surface. The rotation matrix $R_{\mathrm{bn}}$ is found by applying three rotations with Euler angles in the sequence $\Psi, \Theta, \Phi$. 
The resulting velocity in the inertial NED frame is

$$
{ }^{\mathrm{n}} \mathbf{v}^{n b}=R_{\mathrm{bn}}^{T} \cdot{ }^{\mathrm{b}} \mathbf{v}^{n b}
$$

The local (vehicle carried) NED frame always has the same orientation as the fixed frame. Accordingly, the velocity ${ }^{\mathrm{n}} \mathbf{v}^{n b}$ directly describes the motion of the body relative to $\mathcal{F}_{\mathrm{e}}$. Integration of the velocity yields the position relative to the reference point, which can then be transformed to the according ECEF position

$$
{ }^{\mathrm{e}} \mathbf{r}^{e b}={ }^{\mathrm{e}} \mathbf{r}^{e 0}+R_{\mathrm{en}} \cdot{ }^{0} \mathbf{r}^{0 b}
$$

with

$$
R_{\text {en }}=\left[\begin{array}{ccc}
-\sin \left(\varphi_{0}\right) \cos \left(\lambda_{0}\right) & -\sin \left(\lambda_{0}\right) & -\cos \left(\varphi_{0}\right) \cos \left(\lambda_{0}\right) \\
-\sin \left(\varphi_{0}\right) \sin \left(\lambda_{0}\right) & \cos \left(\lambda_{0}\right) & -\cos \left(\varphi_{0}\right) \sin \left(\lambda_{0}\right) \\
\cos \left(\varphi_{0}\right) & 0 & -\sin \left(\varphi_{0}\right)
\end{array}\right]
$$

where $\lambda_{0}$ and $\varphi_{0}$ describe the latitude and longitude of the NED reference point in the earth-centered frame. The nonlinear equations of motion for a rigid body are obtained by combining translational and rotational dynamics. The resulting formulation of Newton-Euler equations is

$$
\begin{aligned}
& \mathrm{b}\left(\frac{\mathrm{d} \mathbf{v}}{\mathrm{d} t}\right)^{n b}=m^{-1}\left({ }^{\mathrm{b}} \mathbf{f}-\boldsymbol{\Omega}^{b} \times m \cdot{ }^{\mathrm{b}} \mathbf{v}^{n b}\right) \\
& \mathrm{b}\left(\frac{\mathrm{d} \boldsymbol{\Omega}}{\mathrm{d} t}\right)^{n b}=\mathbf{I}^{-1}\left({ }^{\mathrm{b}} \mathbf{m}-\boldsymbol{\Omega}^{b} \times \mathbf{I} \boldsymbol{\Omega}^{b}\right)
\end{aligned}
$$

Let ${ }^{\mathrm{b}} \mathbf{f}_{\mathrm{A}} \in \mathbb{R}^{3}$ be the aerodynamic force, ${ }^{\mathrm{b}} \mathbf{f}_{\mathrm{P}} \in \mathbb{R}^{3}$ the propulsive force, ${ }^{\mathrm{b}} \mathbf{f}_{\mathrm{G}}=m g[-\sin \theta \sin \phi \cos \theta \cos \phi \cos \theta]^{T} \in$ $\mathbb{R}^{3}$ the gravitational force with constant gravitational acceleration $g,{ }^{\mathrm{b}} \mathbf{m}_{\mathrm{A}} \in \mathbb{R}^{3}$ the aerodynamic moment, and ${ }^{\mathrm{b}} \mathbf{m}_{\mathrm{P}} \in \mathbb{R}^{3}$ the propulsive moment resolved in $\mathcal{F}_{\mathrm{b}}$. Then, the total forces and moments acting on the aircraft can be expressed as ${ }^{\mathrm{b}} \mathbf{f}={ }^{\mathrm{b}} \mathbf{f}_{\mathrm{A}}+{ }^{\mathrm{b}} \mathbf{f}_{\mathrm{P}}+{ }^{\mathrm{b}} \mathbf{f}_{\mathrm{G}}$ and ${ }^{\mathrm{b}} \mathbf{m}={ }^{\mathrm{b}} \mathbf{m}_{\mathrm{A}}+{ }^{\mathrm{b}} \mathbf{m}_{\mathrm{P}}$, respectively. Modeling the aerodynamic and propulsive contributions will be presented in the following chapters.

The translational (Eq. (7) and rotational equations of motion (Eq. (8)) are defined with respect to the Center of Gravity (CG). Above that, the inertia tensor $\mathbf{I}$ is required for the determination of the rotational motion in the latter equation. As both the position of CG and inertia are a function of the tilt angles $\delta_{W 1}$ and $\delta_{W 2}$, a simple weight and balance estimation is used to incorporate the effect during transition in the model. All relevant components are modeled as simplified mass objects with uniform density. For the Vahana Alpha $\mathrm{f}$, an empty weight of $475 \mathrm{~kg}$ was found while the vehicle can carry a payload of $100 \mathrm{~kg}$, leading to a take-off weight (TOW) of $m=575 \mathrm{~kg}$. In most eVTOL configurations, the battery usually accounts for approximately one third of TOW. According to the Vahana manufacturer, the battery provides a capacity of $38 \mathrm{~kW} \mathrm{~h} \$$ while an energy density at system level of $166.6 \mathrm{~W} \mathrm{~h} \mathrm{~kg}^{-1}$ is extrapolated from the recent development [20], leading to a battery weight of $228.1 \mathrm{~kg}$. For other components, several assumptions were made by the manufacturer during trade studies These include avionics, a crash-rated seat, electrical rudder, and blade pitch actuators, as well as tilt actuators. With experience from helicopter landing skids, the landing gear is assumed to be $2 \%$ of the MTOW. [21] provides a summary of weight equations that allow estimating the weight of the remaining components. These empirical formulations often result from a long history of aircraft manufacturing and do not always consider advancements in lightweight design. In [21], a "technology factor" of 0.8 is applied to all estimations to account for future developments in this field. In the case of the Vahana, a correction factor of 0.415 is required for the weights that the manufacturer does not provide in order to achieve the indicated gross weight. Reducing the weight by $58.5 \%$ seems ambitious. Nevertheless, as no other source of information was accessible, these values are adopted. An overview can be found in Table 1 .

The obtained results for the position of the CG and the inertia tensor are listed below for cruise configuration with $\delta_{W 1}=\delta_{W 2}=0$. The position of CG is expressed in a NED frame centered in a reference point that has its x-location in the nose of the aircraft and its z-location at the point where the skids touch the ground.

https://evtol.news/a3-by-airbus/[Accessed: November 2021]

$\S$ https://wwW.airbus.com/en/innovation/zero-emission/urban-air-mobility/vahana [Accessed: November 2021]

${ }^{q}$ https://acubed.airbus.com/blog/vahana/vahana-configuration-trade-study-part-ii/[Accessed: November 2021] 
Table 1 Estimated component weights, using a correction factor of 0.415 for empirical estimations*.

\begin{tabular}{|c|c|c|}
\hline Component & \# of Components & Assumed Weight in kg \\
\hline \hline Battery & 1 & 228.1 \\
\hline Avionics & 1 & 15 \\
\hline Crash Seat & 1 & 15 \\
\hline Electrical Actuators & 12 & 0.65 \\
\hline Tilt Actuators & 2 & 4 \\
\hline Fuselage & 1 & $17.32^{*}$ \\
\hline Canard & 1 & $25.07^{*}$ \\
\hline Main Wing & 1 & $17.24^{*}$ \\
\hline Vertical Stabilizers & 2 & $1.44^{*}$ \\
\hline Rotor & 8 & $7.91^{*}$ \\
\hline Motor \& Controller & 8 & $5.34^{*}$ \\
\hline Landing Gear & 1 & 32.6 \\
\hline \hline
\end{tabular}

Total Weight

475

$$
\mathbf{r}_{\text {cg }}=\left[\begin{array}{c}
-2.64 \\
0 \\
-1.33
\end{array}\right] \mathrm{m} \quad \mathbf{I}=\left[\begin{array}{ccc}
462 & -44 & -107 \\
-44 & 1080 & 8 \\
-106 & 8 & 1300
\end{array}\right] \mathrm{kg} \mathrm{m}^{2}
$$

The effect of the tilt wings on the Center of Gravity and inertia is shown in Fig. 2. The deviation from cruise condition of all diagonal entries in the inertia tensor stays below $3 \%$ and seems negligible.

The movement of the CG can be approximated in the model as a function of both tilt angles by using a second-order polynomial:

$$
\mathbf{r}_{\mathbf{c g}}=\left[\begin{array}{c}
-2.64 \\
0 \\
-1.33
\end{array}\right]-\left[\begin{array}{c}
9.92 \\
0 \\
90
\end{array}\right] \delta_{W 1} \cdot 10^{-5}-\left[\begin{array}{c}
1.15 \\
0 \\
10
\end{array}\right] \delta_{W 2} \cdot 10^{-4}-\left[\begin{array}{c}
4.45 \\
0 \\
-4.45
\end{array}\right] \delta_{W 1}^{2} \cdot 10^{-6}-\left[\begin{array}{c}
5.14 \\
0 \\
-5.14
\end{array}\right] \delta_{W 2}^{2} \cdot 10^{-6}
$$



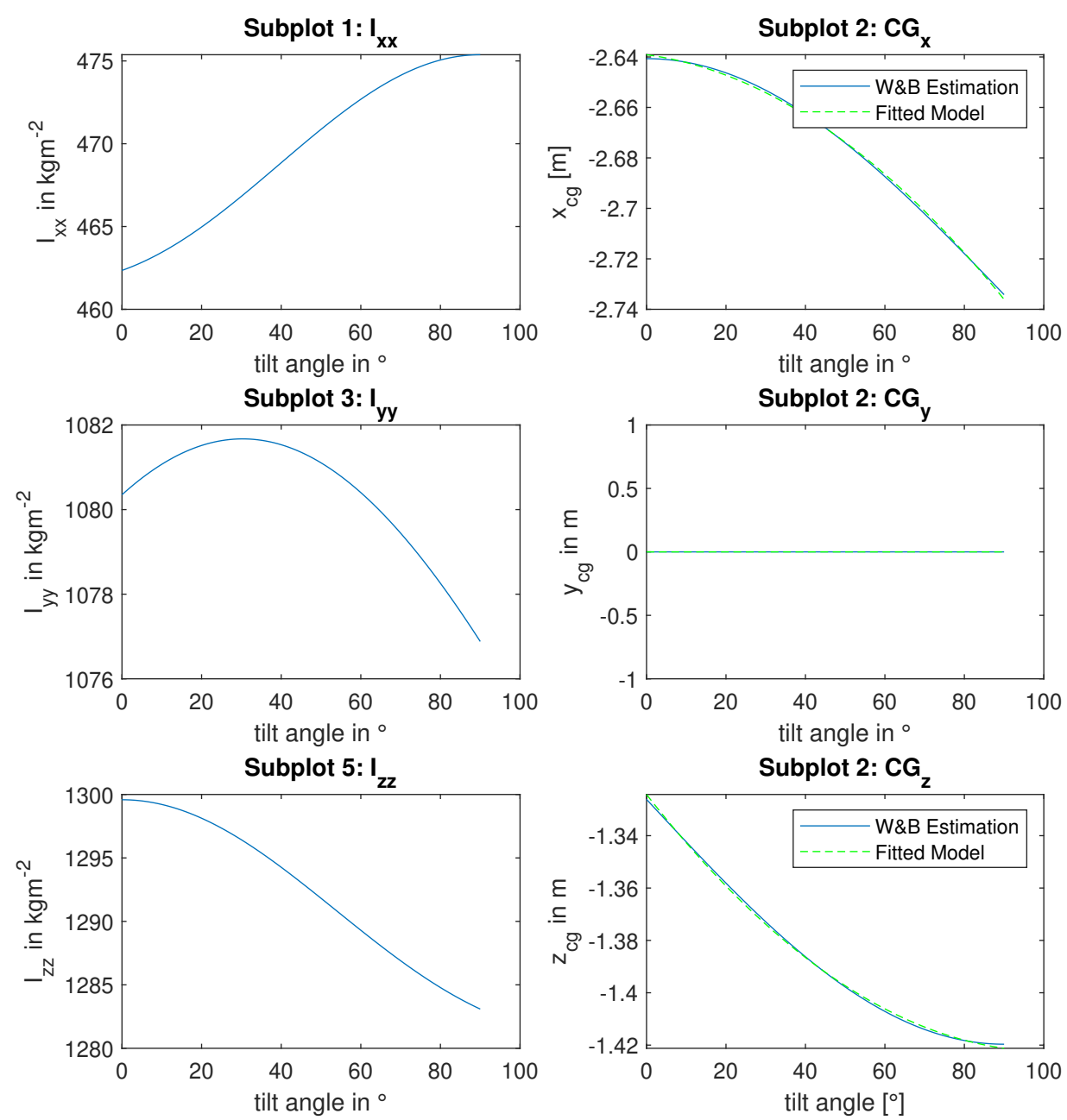

Fig. 2 Effect of tilt angle sweep from $0^{\circ}$ to $90^{\circ}$ where $\delta_{W 1}=\delta_{W 2}$

\section{Aerodynamic Model}

From an aerodynamic point of view, the considered tandem tilt-wing eVTOL is characterized by relatively small dimensions as well as a high number of propulsors and actuators. The latter encompass control surfaces and tilt actuators that transform the vehicle configuration. That leads to a diverse and complex aerodynamic flow field with a high number of independent variables when compared to conventional air- and rotorcraft. As can be seen in Table 2, a total of 28 variables is required to describe the aerodynamic state of the Vahana aircraft. These include all 22 variables representing the aircraft's actuation system, compare isometric view in Fig. 11 and the 6 DoF motion of the body with respect to the surrounding air.

Known vehicle characteristics and effects that drive the aerodynamic complexity are:

- Large flight envelope due to transformation

- Many control surfaces and propulsors

- Propulsion-airframe interactions

- High angle of attack tilt-wing aerodynamics and operations in super-stall regions

- High incidence angle propeller aerodynamics

- Rotor blades with fast-changing rotational speeds and variable blade pitches

- Rotor-wing interaction creating slipstream-induced lift and drag 
- Side-by-side rotor-rotor interactions of near rotors in one plane

- Tandem rotor-rotor interactions of near tandem rotor interactions

Table 2 Independent variables that determine the aerodynamic state.

\begin{tabular}{|c||c|}
\hline Freestream velocity & $\mathbf{v}_{A}^{b}$ \\
Body rates & $\mathbf{\Omega}^{b}$ \\
Tilt angle & $\delta_{W 1}, \delta_{W 2}$ \\
Control surface deflection & $\delta_{\mathrm{A} 1}, \delta_{\mathrm{A} 2}, \delta_{\mathrm{E} 1}, \delta_{\mathrm{E} 2}$ \\
Rotor speed & $\omega_{1} \ldots \omega_{8}$ \\
Blade pitch angle & $\theta_{1} \ldots \theta_{8}$ \\
\hline
\end{tabular}

This seems to be the reason that, despite research efforts in tilt-wing VTOL aircraft and convertiplanes since mid of the $20^{\text {th }}$ century [4, 11, 22, 23], no established aerodynamic model exists. Indeed, propelled by diverse tools for fluid simulation, increased efforts have been made lately to understand the intricate aerodynamics of tilt-wing and multirotors in general [8, 16, 24-26]. In accordance with this trend, finding a representative aerodynamic model can be identified as the most demanding challenge for the work on tilt-wing aircraft, especially from a flight control perspective when considering the transition maneuver.

Aerodynamic modeling for conventional aircraft is usually based on data look-up tables (LUT) or functional representations of non-dimensional aerodynamic coefficients. They depend on the aerodynamic state described by velocity $V$, angle of attack (AoA) $\alpha$, and sideslip angle $\beta$, as well as deflections of aerodynamic control surfaces like the elevator deflection $\delta_{\mathrm{E}}$ or aileron deflection $\delta_{\mathrm{A}}$. These relations are typically derived from analytical approximations, flight experiments, wind tunnel investigations, or computational fluid dynamics (CFD) simulations. Similar analytical and semi-empirical approaches find application for dynamic modeling in the eVTOL field. Pradeep [27] deploys a conventional fixed-wing aerodynamics model for trajectory optimization. Similar analytical approaches with the intent of controller design are found in [28] and [29]. Airfoil data based on 2D analytical methods and wind tunnel investigations are used by Öner et al. [30] for mathematical modeling and vertical control of a tilt-wing. In [31], post-stall characteristics for high incidence angles are added by applying the model developed by Tangler and Ostowari [32]. The original model was developed to describe the post-stall correction of blade-element/momentum predictions of wind turbine blades using wind tunnel data [32, 33]. Post-stall is also considered in [34]. The application of conventional methods for eVTOL modeling is obvious for initial attempts to gain a first insight into system behavior. A short description of the model is given below, while the reader is referred to [35] for a detailed introduction.

A first compromise is required when considering the flow field state. Due to the ability of hover flight, rotorcraft aerodynamics are often described using body-axis translational velocity components $u, v, w$ instead of the aircraft convention $V, \alpha, \beta$ because $\alpha, \beta$ are not defined for $u=0$ or $V=0$. Still, this merely affects a small part of the flight envelope, and most of the relevant formulations and tools make use of the aircraft convention, wherefore this is adopted here. The angle of attack $\alpha$, the sideslip angle $\beta$, and the airspeed $V$ are defined as

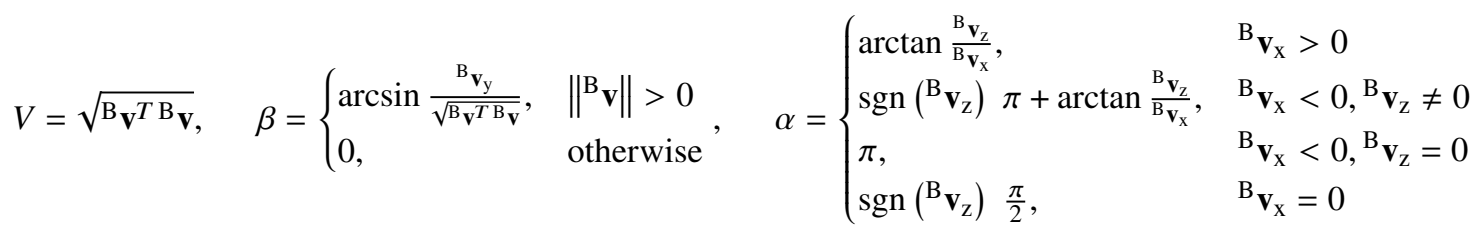

The aerodynamic forces and moments ${ }^{\mathrm{B}} \mathbf{f}_{a}$ and ${ }^{\mathrm{B}} \mathbf{m}_{a}$ are further defined as

$$
{ }^{\mathrm{B}} \mathbf{f}_{\mathrm{a}}=q S{ }^{\mathrm{B}} \mathbf{R}_{\mathrm{W}}\left[\begin{array}{c}
-C_{\mathrm{D}}\left(\alpha_{\mathrm{eff}}, \beta\right) \\
C_{\mathrm{S}}\left(\beta,{ }^{\mathrm{B}} \omega_{\mathrm{z}}\right) \\
-C_{\mathrm{L}}\left(\alpha_{\mathrm{eff}}\right)
\end{array}\right] \quad{ }^{\mathrm{B}} \mathbf{m}_{\mathrm{a}}=q S{ }^{\mathrm{B}} \mathbf{R}_{\mathrm{W}}\left[\begin{array}{c}
b C_{\mathrm{l}}\left(\beta,{ }^{\mathrm{B}} \omega_{\mathrm{x}},{ }^{\mathrm{B}} \omega_{\mathrm{z}}, \delta_{\mathrm{A}}\right) \\
c C_{\mathrm{m}}\left(\alpha_{\mathrm{eff}},{ }^{\mathrm{B}} \omega_{\mathrm{y}}, \delta_{\mathrm{E}}\right) \\
b C_{\mathrm{n}}\left(\beta,{ }^{\mathrm{B}} \omega_{\mathrm{x}},{ }^{\mathrm{B}} \omega_{\mathrm{z}}, \delta_{\mathrm{A}}\right)
\end{array}\right]
$$

with the reference span width of $b=6 \mathrm{~m}$ which includes the vertical stabilizers, average chord length $c=0.67 \mathrm{~m}$, and the resulting reference wing area $S=8.04 \mathrm{~m}$, as well as the dynamic pressure $q=\frac{1}{2} \rho V^{2}$ with $\rho=1.225 \mathrm{~kg} \mathrm{~m}^{-3}$. The 
effective angle of attack is $\alpha_{\text {eff }}=\alpha+\delta_{\text {tilt. }}$. The aerodynamic coefficients can be approximated as

$$
\begin{aligned}
& C_{\mathrm{D}}\left(\alpha_{\mathrm{eff}}, \beta\right)=C_{\mathrm{D}_{0}}+C_{\mathrm{D}_{\alpha}}\left(\alpha_{\mathrm{eff}}\right)+C_{\mathrm{D}_{\beta}}(\beta) \\
& C_{\mathrm{S}}\left(\beta,{ }^{\mathrm{B}} \omega_{\mathrm{z}}\right)=C_{\mathrm{S}_{\beta}} \beta+C_{\mathrm{S}_{\mathrm{r}}}{ }^{\mathrm{B}} \omega_{\mathrm{z}} \\
& C_{\mathrm{L}}\left(\alpha_{\mathrm{eff}}\right)=C_{\mathrm{L}_{0}}+C_{\mathrm{L}_{\alpha}}\left(\alpha_{\mathrm{eff}}\right) \\
& C_{\mathrm{l}}\left(\beta,{ }^{\mathrm{B}} \omega_{\mathrm{x}},{ }^{\mathrm{B}} \omega_{\mathrm{z}}, \delta_{\mathrm{A}}\right)=C_{\mathrm{l}_{\beta}} \beta+C_{\mathrm{l}_{\mathrm{p}}}{ }^{\mathrm{B}} \omega_{\mathrm{x}}+C_{\mathrm{l}_{\mathrm{r}}}{ }^{\mathrm{B}} \omega_{\mathrm{z}}+C_{\mathrm{l}_{\delta_{\mathrm{A}}}} \delta_{\mathrm{A}} \\
& C_{\mathrm{m}}\left(\alpha_{\mathrm{eff}},{ }^{\mathrm{B}} \omega_{\mathrm{y}}, \delta_{\mathrm{E}}\right)=C_{\mathrm{m}_{0}}+C_{\mathrm{m}_{\alpha}}\left(\alpha_{\mathrm{eff}}\right)+C_{\mathrm{m}_{\mathrm{q}}}{ }^{\mathrm{B}} \omega_{\mathrm{y}}+C_{\mathrm{m}_{\delta_{\mathrm{E}}}} \delta_{\mathrm{E}} \\
& C_{\mathrm{n}}\left(\beta,{ }^{\mathrm{B}} \omega_{\mathrm{x}},{ }^{\mathrm{B}} \omega_{\mathrm{z}}, \delta_{\mathrm{A}}\right)=C_{\mathrm{n}_{\beta}} \beta+C_{\mathrm{n}_{\mathrm{p}}}{ }^{\mathrm{B}} \omega_{\mathrm{x}}+C_{\mathrm{n}_{\mathrm{r}}}{ }^{\mathrm{B}} \omega_{\mathrm{z}}+C_{\mathrm{n}_{\delta_{\mathrm{A}}}} \delta_{\mathrm{A}}
\end{aligned}
$$

\begin{tabular}{|c|c|c|c|c|c|}
\hline Variable & Fitted value & Variable & Fitted value & Variable & Fitted value \\
\hline$C_{\mathrm{D}_{\alpha}}(\alpha)$ & $1.06963 \sin (\alpha)^{2}$ & $C_{\mathrm{l}_{\beta}}$ & 0.025 & $C_{\mathrm{m}_{\alpha}}(\alpha)$ & $0.395 \sin (3 \alpha)^{4}+$ \\
\hline$C_{\mathrm{D}_{\beta}}(\beta)$ & $0.108 \beta^{2}$ & $C_{\mathrm{l}_{p}}$ & -0.462 & & $0.5239 \sin (2 \alpha)^{2}$ \\
\hline \multirow[t]{2}{*}{$C_{\mathrm{D}_{0}}$} & 0.08 & $C_{\mathrm{l}_{r}}$ & 0.017 & $C_{\mathrm{m}_{q}}$ & -110.3 \\
\hline & & $C_{1_{\delta_{A}}}$ & -0.165 & $C_{\mathrm{m}_{\delta_{E}}}$ & -3.22 \\
\hline$C_{\mathrm{S}_{\beta}}$ & -0.8 & $C_{\mathrm{n}_{\beta}}$ & -0.06 & $C_{\mathrm{m}_{0}}$ & -0.13 \\
\hline$C_{\mathrm{S}_{r}}$ & 0.469 & $C_{\mathrm{n}_{p}}$ & 0.0366 & & \\
\hline$C_{\mathrm{L}_{\alpha}}(\alpha)$ & $1.71181 \sin (2 \alpha)$ & $C_{\mathrm{n}_{r}}$ & -0.111 & & \\
\hline$C_{\mathrm{L}_{0}}$ & 0.14 & $C_{\mathrm{n}_{\delta_{A}}}$ & 0.015 & & \\
\hline
\end{tabular}

The open-source aerodynamic tool OpenVSP [18], which is based on the vortex lattice method (VLM), is used for the aerodynamic data acquisition. The single coefficients for Eq. [13) are obtained by fitting a polynomial model to the results from OpenVSP, see Table 3 .

Table 3 Fitted aerodynamic parameters from OpenVSP analysis.

With an effective AoA ranging from $0^{\circ}$ to $90^{\circ}$, linear approximations are no longer valid. Therefore, the respective coefficients $C_{\mathrm{L}}, C_{\mathrm{D}}, C_{\mathrm{m}}=f(\alpha)$ are approximated by nonlinear functions to cover the stall and post-stall region, see [35].

The proposed model allows first insights (see Section VI] and applications for flight control design [9]. Still, it neglects or highly simplifies all interactions between aerodynamic components.Using strip theory extends this approach. Strip theory divides the airfoil into several segments, thereby allowing to consider local freestream conditions, including the rotor-induced slipstream [36-38]. The approach presented in [36] is especially useful to rapidly estimate the aerodynamic performance of different configurations. In [39], the authors developed a surrogate model approach for propeller-wing interactions for multiobjective design optimization (MDO). The base model uses the propeller slipstream calculation in combination with a VLM model and drag corrections. The slipstream is also considered by Patterson when comparing different eVTOL configurations [40]. Still, side-by-side and tandem rotor-rotor interactions are not included. While they would have been preferred, simple approximations of the complex aerodynamic interactions have not been identified.

High fidelity investigations are commonly based on either wind tunnel tests [14, 16, 41, 43] or CFD simulations [8, 44]. A combination of the latter is used in [15]. While these approaches promise to gain valuable insights into aerodynamic behavior, they are extremely expensive and have high demands on hardware and infrastructure. As a consequence, the development in the field of DEP aircraft is accompanied by the release of new appropriate tools for their efficient investigation. In [45], the mid-fidelity aerodynamic open-source tool DUST is introduced. DUST is intended for the application of eVTOL and is based on a vortex particle method (VPM) to allow a numerically stable Lagrangian description of the free vorticity evolution. Good conformance with CFD results is shown in [17] when applied to the Airbus $A^{3}$ Vahana. In contrast to CFD, DUST does not require extensive preparation of simulations in the form of meshing. That allows automatic execution of numerical experiments, which is a decisive factor during the exploration of the high-dimensional design space.

To derive mathematical models from real or numerical experiments, usually, a one-factor-at-a-time (OFAT) strategy is used to capture dependencies between single variables [43]. Even when computational efficient mid-fidelity simulations 
like DUST are deployed, this approach would disrupt the timeframe of the planned investigations on flight controls and FDIR. NASA proposed a promising framework called Rapid Aero Modeling (RAM) [16, 46, 47] that allows the derivation of high-fidelity data-driven aerodynamic models. It is deployed successfully for different eVTOL configurations, including tilt-wings [16, 43, 47], and is especially suited to derive models applicable for flight dynamics investigations. The key concept is to drastically reduce the number of experimental design points required to fit a data model while maintaining all relevant effects and relationships between dependent and independent variables. A statistically distributed arrangement of design points in the high-dimensional input space is achieved by utilizing design of experiments (DoE) theory. While the authors in [16, 47] conduct either wind-tunnel experiments or CFD simulations to gather data for a polynomial approximation, the choices of the method for the aerodynamic investigation and model generation are free in general. With regard to the expected medium fidelity and resource efficiency, the presented model will be extended by a data-driven approach inspired by the RAM methodology using DUST as a test facility in the future. It shall be mentioned here that Simmons et al. [16] showed how to merge high fidelity propeller models with the resulting aerodynamic model. Consequently, the rotor model in Section V.C. is not redundant.

\section{Propulsion System Model}

In contrast to fuel-propelled aircraft, eVTOLs merely require electrical connections between motors and energy sources, thereby offering greater flexibility when designing the propulsion system. Distributed electric propulsion (DEP) increases efficiency due to better placement and sizing of single propulsors, improves resilience in case of engine failures, and enables new forms of vehicle control [48]. Especially during hover and transition flight phases, eVTOLs can be considered propulsion-dominated aircraft, meaning that propulsion has the biggest direct impact on force and torque balances and affects the flow field around the vehicle and thereby its aerodynamics, see Section IV] Consequently, the propulsion system is the key element concerning performance and controllability. Since mass and size are crucial parameters, the motor selection is a design decision that significantly impacts aircraft performance [49, 50]. The motor is supplied by DC, given a direct current (DC) power supply realized by batteries or fuel cells and a DC-backbone network. Thus, either a DC motor or an alternating current (AC) motor combined with a DC/AC converter can be used. The latter brings additional complexity and weight to the system. Thus for small systems (e.g., radio-controlled aircraft), a DC motor is the appropriate choice. In contrast, for larger vehicles, a closer distinction has to be made [51, 52].

The dynamic model of a permanent magnet synchronous motor (PMSM) shares many similarities with the brushless DC (BLDC) model. However, a PMSM is used in this model since they seem to dominate in current eVTOL concepts (e.g., Airbus $A^{3}$ Vahana). The following parameters are assumed based on the MAGicALL MAGiDRIVE motor $\llbracket$ The approximated power $P$ is $45 \mathrm{~kW}$, the maximum angular velocity $\omega_{\max }$ is around $4000 \mathrm{rpm}$ to $4500 \mathrm{rpm}$, and the torque is ranges from $45 \mathrm{~N} \mathrm{~m}$ to $200 \mathrm{~N} \mathrm{~m}$. The electric-to-mechanic efficiency $\eta$ is assumed to be approximately $92.5 \%$. PMSMs are fed by AC and controlled using vector control. For further details, see, e.g., [53]-55].

\section{A. Motor Model}

PMSM model In general, an electric motor can be mathematically described via Ohms law, the induction law due to the coil, and the back electromotive force. A change of the flux $\Psi$ induces a voltage. Thus, according to [55, 56], the PMSM is expressed via:

$$
\left[\begin{array}{l}
v_{a} \\
v_{b} \\
v_{c}
\end{array}\right]=\left[\begin{array}{ccc}
R_{a} & 0 & 0 \\
0 & R_{b} & 0 \\
0 & 0 & R_{c}
\end{array}\right]\left[\begin{array}{c}
i_{a} \\
i_{b} \\
i_{c}
\end{array}\right]+\frac{d}{d t}\left[\begin{array}{l}
\Psi_{a} \\
\Psi_{b} \\
\Psi_{c}
\end{array}\right]
$$

with the stator currents $i_{a}, i_{b}$, and $i_{c}$, the stator voltages $v_{a}, v_{b}$, and $v_{c}$, and the stator flux $\Psi_{a}, \Psi_{b}$, and $\Psi_{c}$. The magnetic flux in each coil consists of the current-induced flux from the coils and the magnetic flux from the rotor's permanent magnets $\Psi_{a m}, \Psi_{b m}, \Psi_{c m}$ :

$$
\left[\begin{array}{l}
\Psi_{a} \\
\Psi_{b} \\
\Psi_{c}
\end{array}\right]=\left[\begin{array}{ccc}
L_{a} & L_{a b} & L_{a c} \\
L_{b a} & L_{b} & L_{b c} \\
L_{c a} & L_{c b} & L_{c}
\end{array}\right]\left[\begin{array}{c}
i_{a} \\
i_{b} \\
i_{c}
\end{array}\right]+\left[\begin{array}{l}
\Psi_{a m} \\
\Psi_{b m} \\
\Psi_{c m}
\end{array}\right]
$$

Since the stator is assumed to be symmetrical, $R=R_{a}=R_{b}=R_{c}$ holds for the resistances. The inductance entries are then equal as well and can be substituted by the inductance $L=L_{a}=L_{b}=L_{c}$ and cross-inductance

\footnotetext{
"Data provided at https://acubed.airbus.com and http://www.magicall.biz are used as sources.
} 
$M=L_{a b}=L_{b c}=L_{c a}$. With the condition $i_{a}+i_{b}+i_{c}=0$, the inductance matrix $\mathbf{L}$ can be rewritten as:

$$
\mathbf{L}=\left[\begin{array}{ccc}
L-M & 0 & 0 \\
0 & L-M & 0 \\
0 & 0 & L-M
\end{array}\right]
$$

resulting in the explicit state-space formulation of the electric motor

$$
\frac{d}{d t}\left[\begin{array}{l}
i_{a} \\
i_{b} \\
i_{c}
\end{array}\right]=\left[\begin{array}{ccc}
\frac{1}{L-M} & 0 & 0 \\
0 & \frac{1}{L-M} & 0 \\
0 & 0 & \frac{1}{L-M}
\end{array}\right]\left(\left[\begin{array}{c}
v_{a} \\
v_{b} \\
v_{c}
\end{array}\right]-\left[\begin{array}{ccc}
R & 0 & 0 \\
0 & R & 0 \\
0 & 0 & R
\end{array}\right]\left[\begin{array}{c}
i_{a} \\
i_{b} \\
i_{c}
\end{array}\right]-\frac{d}{d t}\left[\begin{array}{c}
\Psi_{a m} \\
\Psi_{b m} \\
\Psi_{c m}
\end{array}\right]\right)
$$

if the $\mathbf{L}$ does not depend on the time. The resulting motor torque $\tau_{M}$ can be calculated via:

$$
\tau_{M}=\frac{e_{a} i_{a}+e_{b} i_{b}+e_{c} i_{c}}{\omega}
$$

\section{B. Battery Model}

The energy source and batteries exhibit only little relevant dynamics for flight control design. However, the impact of the energy source increases for FDIR investigation and trajectory optimizations. Thus, this section briefly discusses the relevant effects of the battery model.

In [57], the authors investigate different high-power/high-energy battery hybrids on fixed-wing eVTOLs. They emphasize the importance of an optimized energy storage system that is capable of providing a high (peak) power for high-power flight phases while also achieving high energy densities that enable better energy/weight rations and longer endurance.

\section{Rotor Model}

Next to medium fidelity, two ambitions of the presented modeling approach for the rotor are stability and consistency. That means, for example, that it should consider both directions of rotor rotation and negative blade pitch angles as well as to avoid division by zero issues as they occur when the rotor speed $\omega$ approaches zero during an engine failure. The rotor model is chiefly based on a combination of Momentum Theory (MT) and Blade Element Theory (BET) and extended by additional effects.

Next to the operation conditions of a rotor, the freestream velocity vector $\mathbf{v}_{\infty} \in \mathbb{R}^{3}$ has a considerable influence on its aerodynamic behavior. Neglecting the influence of other aerodynamic or propulsive components on the flow field, the effective velocity at the rotor is the sum of the translational aerodynamic velocity of the aircraft center of gravity (CG) with respect to the surrounding air and the Euler term considering the body rotational rate

$$
\mathbf{v}_{\infty}=R_{\mathrm{rb}}\left({ }^{\mathrm{b}} \mathbf{v}_{\mathrm{A}}+\mathbf{\Omega}^{b} \times \mathbf{r}\right)
$$

The different orientation of the rotor frame with respect to the aircraft frame due to a wing tilt-angle $\delta_{\mathrm{W}}$ is reflected in the rotation matrix $R_{\mathrm{rb}}$.

If wind gusts act individually on each rotor, the wind velocity component cannot be incorporated into the body aerodynamic velocity. Instead, the body velocity with respect to the inertial reference frame is complemented by a local gust vector at the position of the rotor in the velocity field.

$$
\begin{gathered}
{ }^{\mathrm{n}} \mathbf{v}_{\text {gust }}=f\left({ }^{\mathrm{n}} \mathbf{r}^{b}+{ }^{\mathrm{n}} \mathbf{r}^{b r}\right) \\
\mathbf{v}_{\infty}=R_{\mathrm{rb}}\left({ }^{\mathrm{b}} \mathbf{v}_{A}+\boldsymbol{\Omega}^{b} \times{ }^{b} \mathbf{r}^{b r}+R_{\text {bn }}{ }^{\mathrm{n}} \mathbf{v}_{\text {gust }}\right)
\end{gathered}
$$

For momentum theory, it is convenient to separate the freestream velocity vector into the in-plane velocity $V_{\mathrm{xy}}$ and the perpendicular rotor inflow $V_{\mathrm{z}}$.

A rotor transfers mechanical rotational power from the shaft to the surrounding fluid, thereby accelerating it [58]. The induced velocity change $v_{\mathrm{i}}$ of the fluid perpendicular to the rotor plane is responsible for generating the thrust $T$ 
according to Newton's third law. After the introduction of induced velocity, the rotor flow state is described in the following by the dimensionless inflow ratio $\lambda$ and advance ratio $\mu$ :

$$
\begin{gathered}
\lambda=\frac{V_{\mathrm{z}}+v_{\mathrm{i}} \cdot \operatorname{sgn}\left(c_{\mathrm{T}}\right)}{V_{\mathrm{t}}} \\
\mu=\frac{V_{\mathrm{xy}}}{V_{\mathrm{t}}}
\end{gathered}
$$

The tip velocity $V_{\mathrm{t}}=|\omega| R$ equals zero when $\omega=0$, wherefore division by zero must be avoided. The direction of the induced velocity is considered to point in the opposite direction of the resulting thrust, considered here by the sign of the thrust coefficient $c_{\mathrm{T}}$ from Eq. 35.

According to [58], the power transferred to the fluid for forward motion of the rotor is the sum of induced and climb power

$$
P_{\mathrm{i}}=\kappa\left(\left|T v_{\mathrm{i}}\right|+T V_{\mathrm{z}}\right)
$$

with empirical loss factor $\kappa$ that accounts for losses like nonuniform inflow. Not all of the shaft power can be exploited for the forward motion. The main share of losses is due to drag forces acting on the rotor blades during the rotational motion [58]. When averaging over the azimuth of the propeller disc, the resulting in-plane force $H$, its according vector form ${ }^{\mathrm{r}} \mathbf{h}_{\mathrm{P}} \in \mathbb{R}^{3}$, and total torque $Q$ can be calculated with the result from Blade Element Theory [58, 59]

$$
\begin{gathered}
H=c_{\mathrm{F}} \cdot \frac{\mu}{4}\left(c_{\mathrm{d} 0}+c_{1 \alpha}\left|\lambda \cdot \theta_{\mathrm{b}}\right|\right) \\
{ }^{\mathrm{r}} \mathbf{h}_{\mathrm{P}}=\frac{1}{V_{\mathrm{xy}}}\left[\begin{array}{c}
v_{\infty, \mathrm{x}} \\
v_{\infty, \mathrm{y}} \\
0
\end{array}\right] \cdot H \\
Q=-\left(c_{\mathrm{F}} \cdot R \frac{c_{\mathrm{d} 0}}{4}\left(1+4.65 \mu^{2}\right)+R(\lambda T+\mu H)\right) \cdot \operatorname{sgn}(\omega)
\end{gathered}
$$

with the blade profile specific coefficients for the drag $c_{\mathrm{d} 0}$ and the lift slope $c_{1 \alpha}$, blade pitch angle $\theta_{\mathrm{b}}$, and the base for the definition of the dimensionless rotor coefficients $c_{\mathrm{F}}=\sigma \rho A_{\mathrm{e}} V_{\mathrm{t}}^{2}$ with effective rotor area $A_{\mathrm{e}}$, see Section V.C.1. The empirical factor 4.65 in Eq. 27) shows better conformance than the theoretical solution, as it incorporates the viscous drag due to span wise flow [59]. That results in the total required power at the propeller:

$$
P_{\text {req }}=Q \cdot \omega
$$

Additional losses, e.g., due to mechanical transmission, can be considered by introducing a degree of efficiency $\eta_{\mathrm{P}}$ for each propulsion unit.

The induced velocity varies according to the freestream conditions and is calculated using MT. In [60], the general formulation is given as

$$
\begin{gathered}
v_{\mathrm{i}}=\frac{v_{\mathrm{h}}^{2}}{\sqrt{V_{\mathrm{xy}}^{2}+\left(V_{\mathrm{z}}+v_{\mathrm{i}}\right)^{2}}} \\
v_{\mathrm{h}}=\sqrt{\frac{|T|}{2 \rho A_{\mathrm{R}}}}
\end{gathered}
$$

where $v_{\mathrm{h}}$ is the required induced velocity to achieve the actual thrust $T$ under hover flight condition. For negative rotor inflows, the opposed directions of freestream and induced velocity result in a recirculation at the rotor tip and cause a turbulent wake [60]. The assumptions made during the calculation of the induced velocity are not valid in the presence of turbulence, and there occurs a singularity in the solution of MT in the range of $-2 v_{\mathrm{h}} \leq V_{\mathrm{c}} \leq 0$ where vortex ring state (VRS) is encountered. Several empirical models exist to estimate the rotor-induced velocity during VRS [58, 60]. Leishman [60] provides a simple approach with a fourth-order polynomial that allows a continuous approximation for the full VRS range

$$
\frac{v_{\mathrm{i}}}{v_{\mathrm{h}}}=\kappa+k_{1}\left(\frac{V_{\mathrm{z}}}{v_{\mathrm{h}}}\right)+k_{2}\left(\frac{V_{\mathrm{z}}}{v_{\mathrm{h}}}\right)^{2}+k_{3}\left(\frac{V_{\mathrm{z}}}{v_{\mathrm{h}}}\right)^{3}+k_{4}\left(\frac{V_{\mathrm{z}}}{v_{\mathrm{h}}}\right)^{4}
$$


where $k_{1}=-1.125, k_{2}=-1.372, k_{3}=-1.718$, and $k_{4}=-0.655$. The original approximation does not account for the effect of the in-plane velocity, which, when high enough, can dissolve the tip vortex without encountering VRS [60]. To consider this effect in the model, the solution from momentum theory is valid in the range of VRS if $V_{\mathrm{xy}}>0.9 \cdot v_{\mathrm{h}}$.

A high fidelity VRS model was proposed by Johnson in [61] based on wind-tunnel test campaigns. It comprises a baseline model that covers the singularity in the MT solution and approximates the turbulent region by an additional VRS model. To deploy it for the dynamic flight simulation, slight changes are introduced into the baseline model. The original third-order polynomial is extended to a fourth-order polynomial to allow a continuous blending on both sides of the singularity (see Figure 3). That reduces issues when using Newton's iterative method to solve the equations for induced velocity and thrust. In addition, the parameter $V_{\mathrm{xC}}$ is changed from 0.75 to 0.8 to allow a smooth transition from MT to the baseline model.

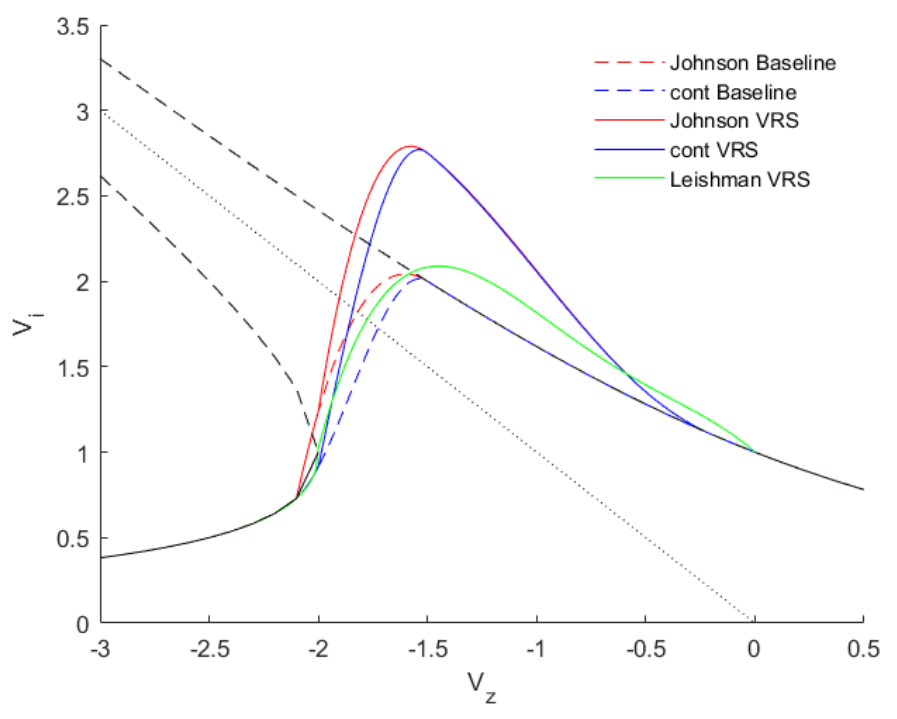

Fig. 3 Comparison of original Johnson [61] and Leishman [60] VRS models and the adapted continuous Baseline and VRS model at $V_{\mathrm{xy}}=0$.

\section{Effective Area}

The high pressure gradient at the blade tip causes the formation of a trailing vortex which decreases the performance of the rotor [60]. In contrast to the idealized momentum theory solution, the lift at the blade tip decreases to zero. This loss can be accounted for by considering an effective rotor area [60]. That also offers the opportunity to consider the proportionate root cutout $r_{0}$ which is given by design. The effective area $A_{\mathrm{e}}$ is defined as [60]

$$
A_{\mathrm{e}}=\pi R_{\mathrm{e}}^{2}=\pi B^{2} R^{2}-\pi r_{0}^{2} R^{2}
$$

where the factor $B$ represents the reduced effective area due to tip losses. A general approximation from [60] for the tip loss is

$$
B=1-\frac{1.386 \lambda}{N_{\mathrm{b}}}
$$

with $N_{\mathrm{b}}$ being the number of blades.

\section{Blade Pitch}

Next to the rotational speed, the Vahana offers to adopt the rotors operating conditions via a variable blade pitch. That is especially useful for tilt-wing vehicles to avoid efficiency losses due to the opposed rotor working states in vertical and horizontal flight. Moreover, the joint deployment of blade pitch and rotor speed supports an efficient control allocation that allows to avoid cross-coupling effects while generating rotor-based steering torques without increasing aerodynamic drag, as conventional aerodynamic rudders do. Other advantages are [60, 62, 63]: 
- Adopt to environmental circumstances

- Increases the spectrum of covered operating points for the motor

- Helps trimming and allows to change the trim point

- Helps to re-trim in case of failure

- Another high-bandwidth control input

When considering a propeller blade profile, the variable blade pitch allows to adjust the angle of attack (AoA) $\alpha$ of the profile with respect to the rotor flow state, defined by $\lambda$ and $\mu$ [64]:

$$
\alpha=\frac{\theta_{\mathrm{b}} \cdot \operatorname{sgn}(\omega)}{3}\left(1+\frac{3}{2} \mu^{2}\right)-\frac{\lambda}{2}
$$

This definition reflects that the same blade pitch angle corresponds to negative angle of attack with reversed direction of rotation. Assuming that the induced angle faced by the blade element is small, the thrust is directly proportional to the lift and consequently to the angle of attack [60]:

$$
c_{\mathrm{T}}=\sigma c_{1 \alpha} \alpha
$$

In order to cover stall effects at high AoA, the thrust coefficient is blended out around $22.5^{\circ}$ :

$$
c_{\mathrm{T}, \mathrm{stall}}=\frac{c_{\mathrm{T}}}{1+\exp \left(-100\left(-\alpha+\frac{\pi}{8}\right)\right.}
$$

The thrust $T$ is obtained using the thrust coefficient found in Eq. (36) and the effective rotor area from Eq. (32).

$$
T=\frac{\rho}{2} c_{\mathrm{T}, \text { stall }} A_{\mathrm{e}} V_{\mathrm{t}}^{2}
$$

The effect of the variable pitch angle on the rotor torque was already covered in Eq. 25 for the rotor $H$-Force.

\section{Ground Effect}

As take-off, transition, and landing phases are of special interest for eVTOL dynamics investigations and general flight missions are expected to be restricted to low altitude bands, the ground effect should be included in the rotor model. In the presence of the ground, the induced velocity in the rotor plane is reduced, implying that hover power close to the ground is lower than far from it [64]. An empiric formulation for the corrected thrust $T_{\mathrm{g}}$ at altitude $z$ above the ground is taken from [65]:

$$
\frac{T_{\mathrm{g}}}{T}=\frac{1}{1-\left(\frac{R}{4|z|}\right)^{2}\left(\frac{1}{1+\left(\frac{|\mathrm{vv}|}{v_{\mathrm{i}}}\right)^{2}}\right)}
$$

where $r$ is the radius of the rotor. As this was researched for helicopter rotors, one should take the radius of the total reference area of a multicopter. According to Eq. (38), the influence of ground effect is negligible if $R /|z|<0.1$. Solutions for $R /|z|>5$ introduce significant errors and should be replaced by the value at the limit $R /|z|=5$.

\section{Blade Flapping}

Blade flapping is a phenomenon caused by the different flow conditions at the rotor blade during one revolution due to advancing and retreating motion relative to the inflow. The differential lift on both sides causes a gyroscopic torque on the rotor plane that tilts the rotor [60]. The resulting tip path flaps up relative to the rotor center when advancing and down when retreating. The angle between rotor plane and blade tip is called flapping angle $\beta$, which is a function of azimuth angle $\Psi$.

Blade flapping has fast dynamics compared to the aircraft's dynamics. It takes about one revolution for the rotor to settle into a new equilibrium state of aerodynamic, centrifugal, and inertial moments [60]. Therefore, blade flapping dynamics can be neglected, and a static model is chosen. The flapping angel $\beta$ is determined by the longitudinal flapping w.r.t. the $x$-axis $\beta_{\mathrm{c}}$ and the lateral flapping w.r.t. the $y$-axis $\beta_{\mathrm{s}}$. Both angles describe how the rotor planes are tilted w.r.t. the original $x-y$ rotor plane. The flapping angle with respect to the azimuthal position is thus [60]

$$
\beta(\psi)=\beta_{0}+\beta_{\mathrm{c}} \cos (\psi)+\beta_{\mathrm{s}} \sin (\psi)
$$


While the thrust component in the vertical direction stays unaffected, there are additional components in lateral and longitudinal direction, summed up into the vector $\Delta_{\text {flap. }}$. From the complex formulation of $\beta_{\mathrm{c}}$ and $\beta_{\mathrm{s}}$ in [66], a simple form is deduced in [67]:

$$
\begin{aligned}
& \beta_{\mathrm{c}}=-\frac{\left|V_{\mathrm{xy}}\right|}{V_{\mathrm{t}}} A_{1 \mathrm{c}}-\frac{1}{\omega} B_{2} \Omega_{1}+\frac{1}{\omega} B_{1} \Omega_{2} \\
& \beta_{\mathrm{s}}=-\frac{\left|V_{\mathrm{xy}}\right|}{V_{\mathrm{t}}} A_{1 \mathrm{~s}}+\frac{1}{\omega} B_{1} \Omega_{1}-\frac{1}{\omega} B_{2} \Omega_{2}
\end{aligned}
$$

resulting in

$$
A_{\text {flap }}=\frac{1}{R}\left[\begin{array}{ccc}
-A_{1 \mathrm{c}} & A_{1 \mathrm{~s}} & 0 \\
-A_{1 \mathrm{~s}} & -A_{1 \mathrm{c}} & 0 \\
0 & 0 & 0
\end{array}\right] \quad B_{\text {flap }}=\left[\begin{array}{ccc}
-B_{2} & B_{1} & 0 \\
B_{1} & -B_{2} & 0 \\
0 & 0 & 0
\end{array}\right]
$$

where $\mathbf{A}_{\text {flap }}$ represents the flapping angle due to translational and $B_{\text {flap }}$ due to rotational motion. This yields the flapping force vector ${ }^{\mathrm{r}} \mathbf{f}_{\text {flap }} \in \mathbb{R}^{3}$ [67]:

$$
{ }^{\mathrm{r}} \mathbf{f}_{\text {flap }}=T\left(A_{\text {flap }} \frac{V_{\text {xy }}}{\omega}+B_{\text {flap }} \frac{\mathbf{\Omega}^{\mathbf{b}}}{\omega}\right)
$$

In [67], the authors propose to derive $\mathbf{A}_{\text {flap }}$ and $\mathbf{B}_{\text {flap }}$ from flight tests. Otherwise, the calculation of these matrices is a complex task [67]. However, the calculation can be drastically simplified by introducing the assumption $\mu^{2} \approx 0$. The calculation of the different matrices can be found in [66]. It shall be mentioned here that the flapping angles $\beta_{\mathrm{c}}$ and $\beta_{\mathrm{s}}$ from Eq. (41) and Eq. (40) are neglected in the calculation of the rotor blade AoA in Eq. (34).

\section{Gyroscopic Torque}

Gyroscopic torque relies on the conservation of rotational momentum l. The rotational momentum of a rotating body, in this case the propeller at rotational speed $\omega$, is perpendicular to the rotating plane. Changing the orientation of that body with angular velocity $\Omega$, here the aircraft rotational rates, generates a torque that is perpendicular to the plane spanned by the rotational velocity $\Omega$ and the rotational momentum vector $\mathbf{l}$, whose magnitude is defined by $\mathbf{l}=I_{\mathrm{R}} \omega$ with $I_{\mathrm{R}}$ being the rotor inertia. That is because the rotational momentum vector is changed by $\Delta \mathbf{l}$, which requires a torque vector in the same direction. According to [67], the gyroscopic torque of the rotor can be expressed as

$$
M_{\mathrm{g}, \mathrm{i}}=-\mathbf{\Omega}^{b} \times I_{\mathrm{R}} \omega
$$

\section{Propulsive Forces and Moments}

In order to apply the propulsive forces and moments to the equations of motion in Eq. (7) and Eq. (8), they are summed and transformed to body coordinates

$$
\begin{gathered}
\left({ }^{\mathrm{r}} \mathbf{f}_{\mathrm{R}}\right)_{\mathrm{i}}=\left[\begin{array}{c}
0 \\
0 \\
-\left(T_{\mathrm{g}}\right)_{\mathrm{i}}
\end{array}\right]+\left({ }^{\mathrm{r}} \mathbf{f}_{\mathrm{flap}}\right)_{\mathrm{i}}+\left({ }^{\mathrm{r}} \mathbf{h}_{\mathrm{P}}\right)_{\mathrm{i}} \\
{ }^{\mathrm{b}} \mathbf{f}_{\mathrm{P}}=\sum_{i=1}^{n_{\text {prop }}}\left(\mathbf{R}_{\mathrm{rb}}^{T}\right)_{\mathrm{i}} \cdot\left({ }^{\mathrm{r}} \mathbf{f}_{\mathrm{R}}\right)_{\mathrm{i}} \\
\left({ }^{\mathrm{r}} \mathbf{m}_{\mathrm{R}}\right)_{\mathrm{i}}=\left[\begin{array}{l}
0 \\
0 \\
Q
\end{array}\right]+\mathbf{m}_{\mathrm{gyro}} \\
{ }^{\mathrm{b}} \mathbf{m}_{\mathrm{p}}=\sum_{i=1}^{n_{\text {prop }}}\left[\left(\mathbf{R}_{\mathrm{rb}}^{T}\right)_{\mathrm{i}} \cdot\left({ }^{\mathrm{r}} \mathbf{m}_{\mathrm{R}}\right)_{\mathrm{i}}+\left({ }^{\mathrm{b}} \mathbf{r}^{b r}\right)_{\mathrm{i}} \times\left(\mathbf{R}_{\mathrm{rb}}^{T}\right)_{\mathrm{i}} \cdot\left({ }^{\mathrm{r}} \mathbf{f}_{\mathrm{R}}\right)_{\mathrm{i}}\right]
\end{gathered}
$$


The transformation between rotor and the body frame corresponds to a rotation matrix around the $y$-Axis with angle $\theta_{\mathrm{W}}+\frac{\pi}{2}+\epsilon$, where $\theta_{\mathrm{W}}$ is the tilt angle of the respective wing and $\epsilon$ is fixed design propulsion inclination with respect to the wing. In case of the Vahana, $\epsilon$ is zero.

\section{Model Analysis}

\section{A. Rotor Analysis}

In order to evaluate the accuracy of the rotor model, it is compared to the results from CFD simulations in Fig. 4 , found in [68]. The authors analysed the performance and wake of the famous Caradonna Tung rotor, that is often used as validation case for CFD-based rotor investigations. Basically, this rotor is characterized by two blades with a NACA0012 profile $\left(c_{1 \alpha}=5.7, c_{d 0}=0.02\right)$ and a parameterized rotor radius of $13.67 c$. In the simulation, the rotor

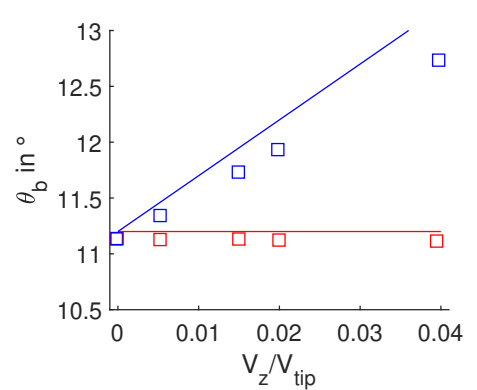

(a)

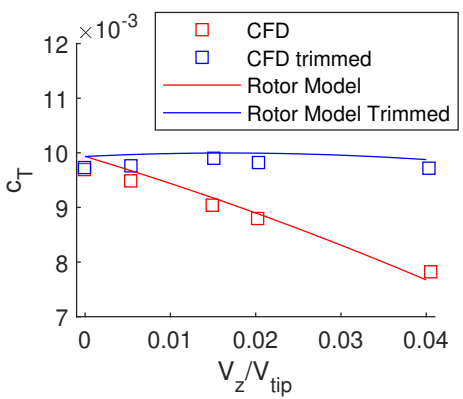

(b)

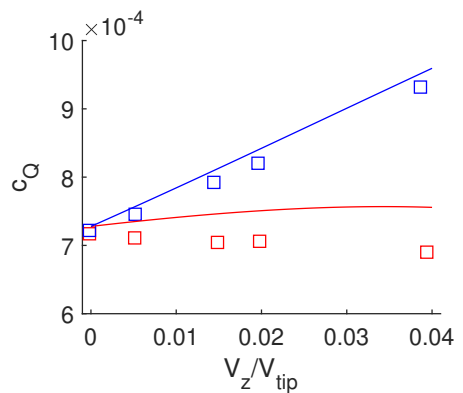

(c)

Fig. 4 Comparison of the rotor model with CFD references of a Caradonna Tung rotor in vertical ascent [68]

performs a vertical ascent, starting at $V_{\mathrm{z}}=0$ until a climb speed of $V_{\mathrm{z}} / V_{\text {tip }}=0.04$ is reached. During ascent, no VRS is encountered and momentum theory is valid. In the first simulation run, the blade pitch angle stays constant (red curves), while in the second it is trimmed (blue curves) to keep the thrust constant despite the change of inflow. The results of both runs show conformance with the CFD references. A slightly steeper gradient for the blade pitch angle is required to trim a constant thrust. This means that the model does not overestimate the rotor performance. For the untrimmed flight, the predicted torque is higher than the CFD reference. The reference torque coefficient shows a slight decreasing trend for rising climb velocities. According to Eq. (27), that means that in the CFD simulation, the non-dimensional inflow $\lambda$ rises slower than the thrust $T$ decreases during climb, in contrast to the model output. Again, this shows a conservative estimation of the presented model, which is preferred.

Next to accuracy, one aim of the modelling approach was to allow counter-rotating rotors. Figure 5 shows the results for two rotors with reversed directions of rotations and blade pitch angles. As expected, both rotors deliver the same thrust while the torques counter one other and sum to zero. That is required for a stable vehicle motion. The plots also

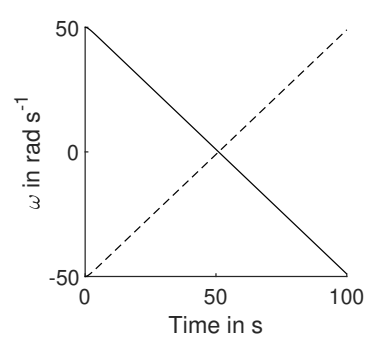

(a)

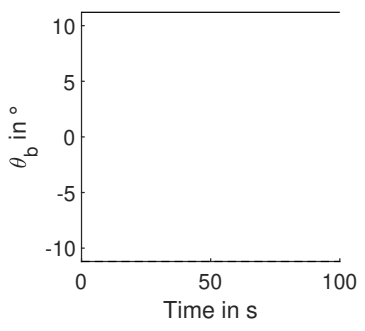

(b)

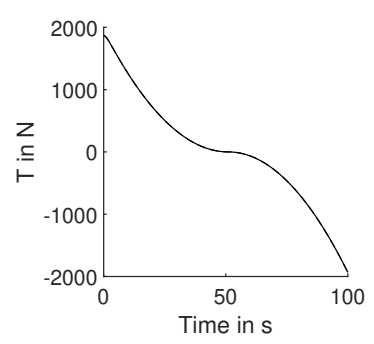

(c)

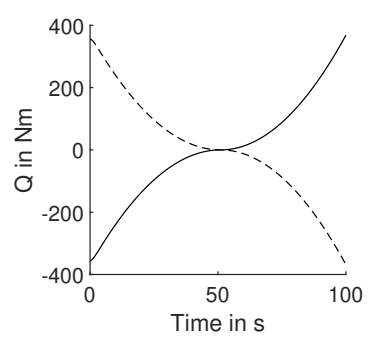

(d)

Fig. 5 Comparison of two counter-rotating rotors with reversed pitch angle $t$ het $a_{\mathrm{b}}$

prove the ability to reverse the direction of rotation, passing a rotational speed of zero, as required. 


\section{B. Trim Study}

A trim point represents the combination of control input values that, under specific conditions, provide an equilibrium of the flight state. In particular, certain derivatives like acceleration and body rates equal zero. In a trim study, stable trim points are calculated under different constraints. Calculating these equilibrium points provides an overview of the system and possible, stable operating points.

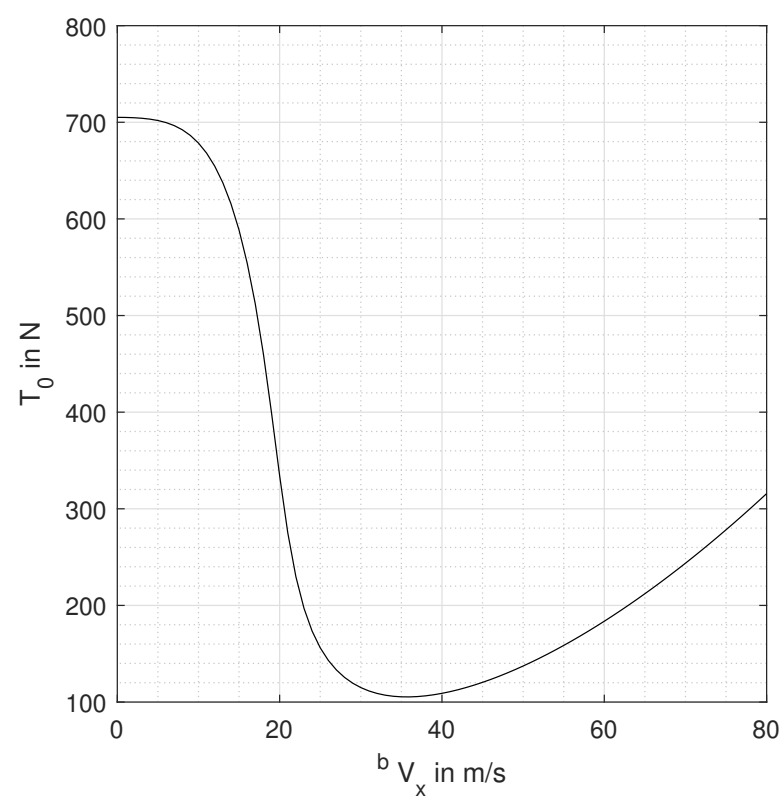

(a)

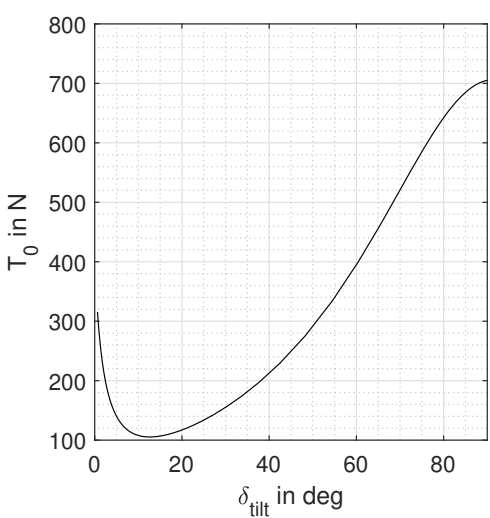

(c)

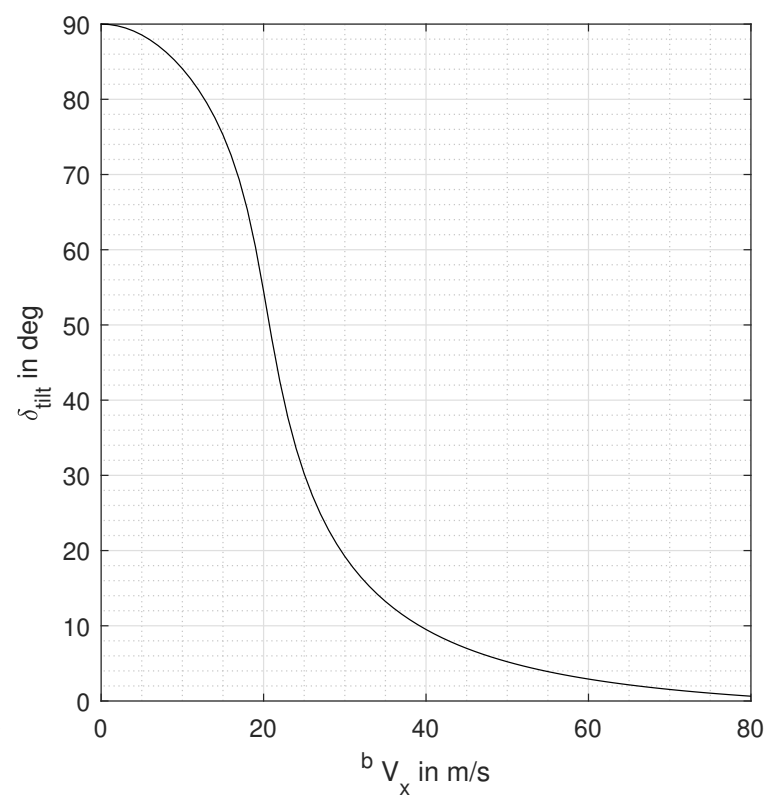

(b)

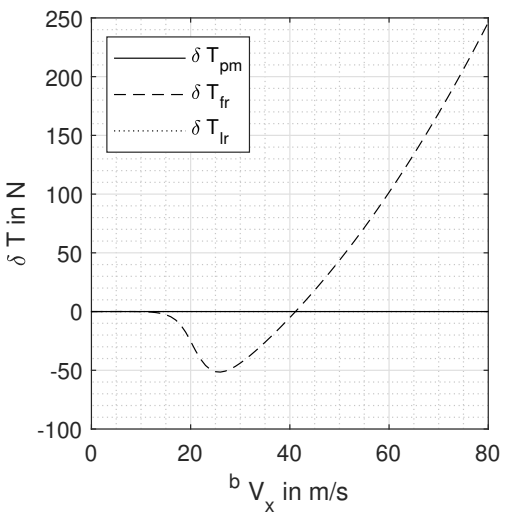

(e)

Fig. 6 Trajectories of a trim study for the transition maneuver of the Vahana aircraft, trimmed at $\alpha=0^{\circ}$ with $\delta_{\text {tilt }}=\delta_{\mathrm{W} 1}=\delta_{\mathrm{W} 2}$.

Figure 6 shows the results of the trim study for the transition maneuver. Although both tilt angles are not coupled, it is assumed here that $\delta_{\text {tilt }}=\delta_{\mathrm{W} 1}=\delta_{\mathrm{W} 2}$. The tilt angle ranges from $0^{\circ}$ for cruise configuration to $90^{\circ}$ for hover configuration. The results show that the vehicle has a trim point for every velocity ${ }^{\mathrm{B}} \mathbf{v}_{x} \in\left[0 \mathrm{~m} \mathrm{~s}^{-1} ; 80 \mathrm{~m} \mathrm{~s}^{-1}\right]$. The resulting stable trajectory is smooth, indicating that a transition from hover to cruise flight is smoothly possible.

During the trimmed transition, a force equilibrium has to be found for weight, propulsive and aerodynamic forces. With a trimmed AoA of zero, the aerodynamics is merely a function of the free stream velocity $V={ }^{\mathrm{b}} V_{\mathrm{x}}$, while the weight force is constant. Figure 6(c) shows the required thrust per rotor $T_{0}$ during transition. The thrust starts at a value of one-eighth of the gravitational force in hover condition. The aerodynamic forces are proportional to the square of the velocity. Initially, that leads to a drop of required thrust, as a high lifting force provided by the airfoils supports the 
vertical force balance. The optimum for cruise efficiency is found at ${ }^{b} V_{\mathrm{x}}=35 \mathrm{~m} \mathrm{~s}^{-1}$ with a corresponding tilt angle of $13^{\circ}$, see also Fig. 6(c) It must be stressed here that the AoA is zero, leading to a relatively small lift force that needs to be supported by a vertical propulsive component over the whole range of ${ }^{\mathrm{B}} \mathbf{v}_{x}$. It can be assumed that, at least above the cruise flight speed of $52.78 \mathrm{~m} \mathrm{~s}^{-1}$ provided by the manufacturer, the tilt angle will be zero because the tilt-wing actuator might not be intended to operate constantly at angles unequal $0^{\circ}$ or $90^{\circ}$. However, as can be seen in Fig. 6(a) more thrust is required at higher speeds to overcome the increased drag force.

The torque balance for the pitch angle can either be trimmed by an elevator deflection $\delta_{\mathrm{E}}$, see Fig. 6(d), or a differential thrust between front and rear rotors $\delta T_{\text {fr }}$, see Fig. 6(e) The trim study shows no difference between both options in the plots Fig. 6(a) - Fig. 6(c) Obviously, an elevator deflection would induce a higher drag force, but that is not considered in Eq. 13a) of the aerodynamic model. In the future, an optimized allocation for efficient pitch angle trimming will be conducted, deploying a combination of elevator deflection and differential thrust using the updated aerodynamic model.

In [69], the authors make a trim study for a small single tilt-wing UAV. The results obtained in a wind tunnel test campaign look qualitatively similar to the ones shown in Fig. 6, although the tilt angle over velocity plot in [69] shows a less pronounced s-shape. The elevator deflections that were used for pitch angle trimming are not comparable due to differences in the torque balances of tandem and single tilt-wing configuration.

\section{Conclusion}

In this paper, we presented an initial mid-fidelity modelling approach of a tandem tilt-wing eVTOL for future flight control and FDIR development. Both formulations for kinematics and propulsion system seem to offer the intended accuracy. The rotor model coincides with simple CFD reference cases, covers dominant effects and prevents limitations of the propeller working state, which were seen in other models. The aerodynamic model follows the conventional coefficient-based approach for aircraft. While giving a first estimate of vehicle dynamics, relevant interactional effects are not considered and should be included in the future. For that, a data-model derived from fluid simulation in DUST has been identified as a promising and efficient approach. Nevertheless, the presented model allows insights into the system behavior of the aircraft. It was shown that the transition trajectory proceeds smoothly along trim points, equally for elevator and differential thrust trimming. Additionally, a qualitative conformance with a trim trajectory derived from wind tunnel tests for a single tilt wing was achieved. Based on the model with updated aerodynamic, an in-depth analysis of the Vahana aircraft is planned in the future, also considering an efficient control allocation for trimming. The model presented in this paper was already deployed as basis for the development of a unified eVTOL control framework. A deeper understanding of the system behavior will support future research in this field.

\section{References}

[1] Cornet, A., Mohr, D., Weig, F., Zerlin, B., and Hein, A.-P., "Mobility of the future: Opportunities for automotive OEMs," , 2012.

[2] Porsche, L., Steinführer, A., and Sondermann, M., "Kleinstadtforschung in Deutschland - Stand, Perspektiven und Empfehlungen," , 2019.

[3] Mattioli, G., and Colleoni, M., “Transport Disadvantage, Car Dependence and Urban Form,”, 2016.

[4] Vieira, D. R., Silva, D., and Bravo, A., "Electric VTOL aircraft: the future of urban air mobility (background, advantages and challenges)," International Journal of Sustainable Aviation, Vol. 5, No. 2, 2019, p. 101. https://doi.org/10.1504/IJSA.2019. 10023187

[5] Hartmann, P., Meyer, C., and Moormann, D., "Unified Velocity Control and Flight State Transition of Unmanned Tilt-Wing Aircraft," Journal of Guidance, Control, and Dynamics, Vol. 40, No. 40, 2017, pp. 1348-1359. https://doi.org/10.2514/1. G002168

[6] Droandi, G., Zanotti, A., and Gibertini, G., "Aerodynamic Interaction between Rotor and Tilting Wing in Hovering Flight Condition," Journal of the American Helicopter Society, Vol. 60, No. 60, 2015, pp. 1-20. https://doi.org/10.4050/JAHS.60. 042011

[7] Rutherford, J. W., and Bass, S. (eds.), Advanced Technology Tilt Wing Study, AIAA 1992-4237, 1992. https://doi.org/10.2514/6. 1992-4237 
[8] Wilke, G. A. (ed.), Aerodynamic Performance of Two eVTOL Concepts, 2018.

[9] Milz, D., and Looye, G., "Tilt-Wing Control Design for a Unified Control Concept," AIAA SCITECH 2022 Forum, 2022. https://doi.org/10.2514/6.2022-1084

[10] UBER Elevate, "Fast-Forwarding to a Future of On-Demand Urban Air Transportation,", 2016.

[11] Pegg, R. J., “Summary of Flight-Test Results of the VZ-2 Tilt-Wing Aircraft,” Technical note, 1962.

[12] Sato, M., and Muraoka, K. (eds.), Flight Test Verification of Flight Controller for Quad Tilt Wing Unmanned Aerial Vehicle, 2013. https://doi.org/10.2514/6.2013-5100

[13] Muraoka, K., Okada, N., Kubo, D., and Sato, M. (eds.), Transition Flight of Quad Tilt Wing VTOL UAV, 2012.

[14] Holsten, J., Ostermann, T., Dobrev, Y., and Moormann, D. (eds.), Model validation of a tiltwing uav in transition phase applying windtunnel investigations, Vol. 28, 2012.

[15] Cetinsoy, E., Dikyar, S., Hancer, C., Oner, K. T., Sirimoglu, E., Unel, M., and Aksit, M. F., "Design and construction of a novel quad tilt-wing UAV," Mechatronics, Vol. 22, No. 6, 2012, pp. 723-745. https://doi.org/10.1016/j.mechatronics.2012.03.003

[16] Simmons, B. M., and Murphy, P. C. (eds.), Wind Tunnel-Based Aerodynamic Model Identification for a Tilt-Wing, Distributed Electric Propulsion Aircraft, 2021. https://doi.org/10.2514/6.2021-1298

[17] Montagnani, D., Tugnoli, M., Zanotti, A., Syal, M., and Droandi, G. (eds.), Analysis of the Interactional Aerodynamics of the Vahana eVTOL Using a Medium Fidelity Open Source Tool, 2020.

[18] McDonald, R. (ed.), Advanced modeling in openvsp, 2016. https://doi.org/10.2514/6.2016-3282

[19] Stengel, R. F., Flight dynamics, Princeton University Press, Princeton NJ, 2004.

[20] Löbberding, H., Wessel, S., Offermanns, C., Kehrer, M., Rother, J., Heimes, H., and Kampker, A., "From Cell to Battery System in BEVs: Analysis of System Packing Efficiency and Cell Types," World Electric Vehicle Journal, Vol. 11, No. 4, 2020, p. 77. https://doi.org/10.3390/wevj11040077.

[21] Kadhiresan, A. R., and Duffy, M. J. (eds.), Conceptual Design and Mission Analysis for eVTOL Urban Air Mobility Flight Vehicle Configurations, 2019. https://doi.org/10.2514/6.2019-2873

[22] Tosti, L. P., "Flight Investigation of the Stability and Control Characteristics of a 1/4-Scale Model of a Tilt-Wing Vertical-TakeOff-and-Landing Aircraft,”, 1959.

[23] McKinney, M. O., Dynamic stability and control characteristics of a cascade-wing vertically rising airplane model in take-offs, landings, and hovering flight, National Advisory Committee for Aeronautics technical note, Vol. 3198, National Advisory Committee for Aeronautics, Washington, D.C., 1954.

[24] Aref, P., Ghoreyshi, M., Jirasek, A., Satchell, M., and Bergeron, K., "Computational Study of Propeller-Wing Aerodynamic Interaction," Aerospace, Vol. 5, No. 5, 2018, p. 79. https://doi.org/10.3390/aerospace5030079

[25] Zanotti, A., Savino, A., Palazzi, M., Tugnoli, M., and Muscarello, V., "Assessment of a Mid-Fidelity Numerical Approach for the Investigation of Tiltrotor Aerodynamics,” Applied Sciences, Vol. 11, No. 8, 2021, p. 3385. https://doi.org/10.3390/app11083385

[26] Droandi, G., Syal, M., and Bower, G. (eds.), Tiltwing multi-rotor aerodynamic modeling in hover, transition and cruise flight conditions, 2018.

[27] Pradeep, P., and Wei, P. (eds.), Energy Optimal Speed Profile for Arrival of Tandem Tilt-Wing eVTOL Aircraft with RTA Constraint, IEEE, 2018. https://doi.org/10.1109/GNCC42960.2018.9018748.

[28] Small, E., and Fresk, E. (eds.), Modelling and Control of a Tilt-Wing Unmanned Aerial Vehicle: June 21-24, 2016, Divani Caravel Hotel, Athens, Greece, IEEE, 2016. URL http://ieeexplore.ieee.org/servlet/opac?punumber=7527573

[29] Haviland, S. T., Bershadsky, D., and Johnson, E. N. (eds.), Dynamic Modeling and Analysis of a VTOL Freewing Concept, 2016. https://doi.org/10.2514/6.2016-1289

[30] Öner, K. T., Çetinsoy, E., Sirimoglu, E. F., Hançer, C., Ünel, M., Akşit, M. F., Gülez, K., and Kandemir, I., "Mathematical modeling and vertical flight control of a tilt-wing UAV," Turk J Elec Eng \& Comp Sci, Vol. 20, No. 20, 2012 , pp. $149-157$. https://doi.org/10.3906/elk-1007-624 
[31] Chauhan, S. S., and Martins, J. R. R. A., “Tilt-Wing eVTOL Takeoff Trajectory Optimization,” Journal of Aircraft, Vol. 57, No. 1, 2020, pp. 93-112. https://doi.org/10.2514/1.C035476.

[32] Tangler, J. L., and Ostowari, C. (eds.), Horizontal axis wind turbine post stall airfoil characteristics synthesization, 1991. URL https://www.osti.gov/biblio/5639513

[33] Viterna, L. A., and Corrigan, R. D., "Fixed pitch rotor performance of large horizontal axis wind turbines,”, 1982.

[34] Rohr, D., Stastny, T., Verling, S., and Siegwart, R., "Attitude and Cruise Control of a VTOL Tiltwing UAV," IEEE Robotics and Automation Letters, Vol. 4, No. 4, 2019, pp. 2683-2690. https://doi.org/10.1109/LRA.2019.2914340

[35] Milz, D., "Design and Evaluation of a Unified Control Framework for Electric Vertical Take-off and Landing Vehicles," Master's thesis, Technical University of Munich, 2020.

[36] Cook, J. (ed.), A Strip Theory Approach to Dynamic Modeling of eVTOL Aircraft, 2021. https://doi.org/10.2514/6.2021-1720.

[37] Sobiesiak, L. A., Fortier-Topping, H., Beaudette, D., Bolduc-Teasdale, F., de de Lafontaine, J., Nagaty, A., Neveu, D., and Rancourt, D. (eds.), Modelling and Control of Transition Flight of an eVTOL Tandem Tilt-Wing Aircraft, 2019. https://doi.org/10.13009/EUCASS2019-137

[38] Jeong, J., Yoon, S., Kim, S.-K., and Suk, J. (eds.), Dynamic Modeling and Analysis of a Single Tilt-Wing Unmanned Aerial Vehicle, 2015. https://doi.org/10.2514/6.2015-1804

[39] Alba, C., Elham, A., German, B., and Veldhuis, L. L. (eds.), A Surrogate-Based Multi-Disciplinary Design Optimization Framework Exploiting Wing-Propeller Interaction, 2017. https://doi.org/10.2514/6.2017-4329

[40] Patterson, M. D., Derlaga, J. M., and Borer, N. K. (eds.), High-Lift Propeller System Configuration Selection for NASA's SCEPTOR Distributed Electric Propulsion Flight Demonstrator, 2016. https://doi.org/10.2514/6.2016-3922

[41] Yanguo, S., and Huanjin, W., "Design of flight control system for a small unmanned tilt rotor aircraft," Chinese Journal of Aeronautics, Vol. 22, No. 3, 2009, pp. 250-256. https://doi.org/10.1016/S1000-9361(08)60095-3

[42] Rothhaar, P. M. (ed.), NASA Langley Distributed Propulsion VTOL Tilt-Wing Aircraft Testing, Modeling, Simulation, Control, and Flight Test Development, 2014. https://doi.org/10.1136/neurintsurg-2020-SNIS.intro

[43] Busan, R. C., Rothhaar, P. M., Croom, M. A., Murphy, P. C., Grafton, S. B., and O'Neal, A. W. (eds.), Enabling Advanced Wind-Tunnel Research Methods Using the NASA Langley 12-Foot Low Speed Tunnel, 2014. https://doi.org/10.2514/6.2014-3000

[44] Deere, K. A., Viken, S., Carter, M., Viken, J. K., Derlaga, J. M., and Stoll, A. M. (eds.), Comparison of High-Fidelity Computational Tools for Wing Design of a Distributed Electric Propulsion Aircraft, 2017. https://doi.org/10.2514/6.2017-3925

[45] Tugnoli, M., Montagnani, D., and Fonte, F. (eds.), Mid-Fidelity Analysis of Unsteady Interactional Aerodynamics of Complex VTOL Configurations, 2019.

[46] Murphy, P. C., and Buning, Pieter G., Simmons, Benjamin M. (eds.), Rapid Aero Modeling for Urban Air Mobility Aircraft in Computational Experiments, 2021. https://doi.org/10.2514/6.2021-1002

[47] Murphy, P. C., Hatke, D., Aubuchon, V. V., Weinstein, R., and Busan, R. C. (eds.), Preliminary Steps in Developing Rapid Aero Modeling Technology, 2020. https://doi.org/10.2514/6.2020-0764

[48] Kim, H. D., Perry, A. T., and Ansell, P. J. (eds.), A Review of Distributed Electric Propulsion Concepts for Air Vehicle Technology, 2018. https://doi.org/10.2514/6.2018-4998

[49] Duffy, M. J., Sevier, A. E., Hupp, R., Perdomo, E., and Wakayama, S. (eds.), Propulsion Scaling Methods in the Era of Electric Flight, 2018. https://doi.org/10.2514/6.2018-4978

[50] Finger, D. F., Braun, C., and Bil, C., "Impact of electric propulsion technology and mission requirements on the performance of VTOL UAVs," CEAS Aeronautical Journal, Vol. 10, No. 3, 2019, pp. 827-843. https://doi.org/10.1007/s13272-018-0352-x

[51] Thurlbeck, A., and Cao, Y. (eds.), Analysis and modeling of uav power system architectures, 2019.

[52] Brelje, B. J., and Martins, J. R. R. A., "Electric, hybrid, and turboelectric fixed-wing aircraft: A review of concepts, models, and design approaches," Progress in Aerospace Sciences, Vol. 104, 2019, pp. 1-19. https://doi.org/10.1016/j.paerosci.2018.06.004.

[53] Binder, A., “Elektrische maschinen und antriebe,”, 2012. https://doi.org/10.1007/978-3-662-53543-1 
[54] Krishnan, R., Electric motor drives: Modeling, analysis, and control, Prentice Hall, 2001.

[55] Krishnan, R., Permanent magnet synchronous and brushless dc motor drives, CRC Press, 2017.

[56] Kundur, P., Balu, N. J., and Lauby, M. G., Power system stability and control, EPRI power system engineering series, McGraw-Hill Education, 1994.

[57] Stahl, P., Roessler, C., and Hornung, M. (eds.), Modelling and Performance Impact of High Power/High Energy Battery Hybrids on Fixed-Wing eVTOL UAV, 2020.

[58] Johnson, W., Helicopter theory, rev. ed. ed., Dover Publications, New York, N.Y., 1994.

[59] Richards, R., "Principles of Helicopter Performance,", 1968.

[60] Leishman, J. G., Principles of helicopter aerodynamics, $2^{\text {nd }}$ ed., Cambridge aerospace series (Cambridge University Press), Vol. 12, Cambridge University Press, Cambridge, 2006.

[61] Johnson, W. (ed.), Model for Vortex Ring State Influence on Rotorcraft Flight Dynamics, 2004.

[62] Arellano-Quintana, V., Merchan-Cruz, E., and Franchi, A., "A novel experimental model and a drag-optimal allocation method for variable-pitch propellers in multirotors," IEEE Access, Vol. 6, 2018, pp. 68155-68168. https://doi.org/10.1109/ACCESS. 2018.2879636

[63] Gupta, N., Kothari, M., et al. (eds.), Flight dynamics and nonlinear control design for variable-pitch quadrotors, 2016.

[64] Seddon, J. M., and Newman, S., Basic helicopter aerodynamics, $3^{\text {rd }}$ ed., Aerospace series, Wiley, Chichester West Sussex UK and Hoboken N.J., 2011.

[65] Cheeseman, I. C., and Bennett, W. E., “The Effect of Ground on a Helicopter Rotor in forward Flight,”, 1955.

[66] Pounds, P. E. I., "Design, construction and control of a large quadrotor micro air vehicle," Ph.D. thesis, The Australian National University, Canberra, Australien, 2007. https://doi.org/10.25911/5D651066AC8BD

[67] Bangura, M., and Mahony, Robert Die interne Fehlermeldung lautet: Die angegebene Datei konnte nicht gefunden werden. (eds.), Nonlinear Dynamic Modeling for High Performance Control of a Quadcopter, 2012.

[68] Nik Mohd, N. A. R., and Barakos, G., "Performance and Wake Analysis of Rotors in Axial Flight Using Computational Fluid Dynamics," Journal of Aerospace Technology and Management, Vol. 9, No. 2, 2017, pp. 193-202. https://doi.org/10.5028/jatm. v9i2.623.

[69] Hartmann, P., Schütt, M., and Moormann, D. (eds.), Konzept eines stetigen bahnreglers für den vollständigen flugbereich eines kippflügelflugzeuges, 2014. 